\title{
Correlation of Chronic Smoking with Meibomian Gland Dysfunction - A Study at Wardha, Maharashtra, India
}

\author{
Swapneel Mathurkar¹, Sachin Daigavane², Madhumita Prasad³, Kervi Mehta ${ }^{4}$ \\ 1, 2, 4 Department of Ophthalmology, Jawaharlal Nehru Medical College, Sawangi, Wardha, Maharashtra, \\ India. ${ }^{3}$ Department of Ophthalmology, Sankara Eye Centre, Indore, Madhya Pradesh, India.
}

\section{ABSTRACT}

\section{BACKGROUND}

Meibomian gland dysfunction (MGD) is one of the causes of evaporative dry eye disease. It is the terminal duct obstruction of the Meibomian gland and is associated with glandular secretion changes. These changes lead to decreased amount of lipids secretion which accounts for instability of tear film leading to evaporative dry eye disease. Chronic smoking also causes irritative, burning eyes along with unstable tear film. We wanted to study the corelation of chronic smoking with Meibomian gland dysfunction.

\section{METHODS}

This is a hospital based observational cross-sectional study that enrolled a total of 100 subjects having Meibomian gland disease (MGD), out of whom 61 were smokers and 39 were non-smokers. All enrolled subjects underwent tear film breakup time (TBUT), Schirmer I test (SIT) and slit-lamp microscope examination of lid margin abnormalities, Meibomian gland expression as well as meibum.

\section{RESULTS}

Our study found that the patients with Meibomian gland dysfunction with the history of chronic smoking had a remarkably decreased value of tear film break up time (TBUT), Schirmer's 1 Test which explains the dry eye symptoms as compared to MGD patients without smoking. No significant differences were seen in lid margin irregularity and meibum secretion. Meibomitis is found in 29 smokers with MGD and 5 non-smokers with MGD which is not significant.

\section{CONCLUSIONS}

Chronic smoking is associated with MGD.

\section{KEY WORDS}

Cigarette Smoking, Meibomian Gland Dysfunction, Tear Film Tests
Corresponding Author: Dr. Kervi Mehta,

Plot No 1, Ratitalai Main Road, Near Nayak Hospital Banswara, Rajasthan 327001, udaipur, India. E-mail:mehta.kervi@gmail.com

DOI: $10.14260 /$ jemds/2021/292

How to Cite This Article:

Mathurkar S, Daigavane S, Prasad M, et al. Correlation of chronic smoking with meibomian gland dysfunction - a study at Wardha, Maharashtra, India. J Evolution Med Dent Sci 2021;10(19):1382-1386, DOI: 10.14260/jemds/2021/292

Submission 03-01-2021,

Peer Review 09-03-2021, Acceptance 16-03-2021, Published 00-05-2021.

Copyright (c) 2021 Swapneel Mathurkar et al. This is an open access article distributed under Creative Commons Attribution License [Attribution 4.0 International (CC BY 4.0)] 


\section{BACKGROUND}

Smoking tobacco is one of the most irresistible addictions, and it has many devastating effects on cardiovascular system, respiratory system and ocular diseases. ${ }^{1-3}$ Because of its addictive habit, it is considered as a serious health problem around the world. Smoking has many adverse ocular side effects and is associated with many ophthalmological disorders like glaucoma, age related macular degeneration, optic neuritis, ocular inflammation.4-6 The interrelation between smoking and ocular diseases continues to progress.

Cigarette smoking causes irritative eyes, burning, itching, irritation and stinging. All these symptoms occur because of effects of cigarette smoking on the corneal tear film. The lipid layer secreted from Meibomian gland, which prevents the evaporation of aqueous layer of tear film. This layer provides lubrication of the ocular surface. Meibomian glands are the holocrine sebaceous glands that are present in the tarsal plate of both the eyes on upper and lower eyelid.

Meibomian gland dysfunction (MGD) is a common eyelid disorder associated with cigarette smoking and its prevalence is $39-50 \%$ in US population and the incidence increases with the increasing age.7,8 It is an ocular surface disease with chronic and diffuse abnormality of the Meibomian glands, which is characterized by terminal duct obstruction and / or qualitative / quantitative changes in the glandular secretion.

It is one of the major causes of evaporative dry eye disease (EDED) ${ }^{9}$ with the growth of epithelial cells of glands which results in blockage of the ducts of the meibomian glands. Blockage leads to decreased lipid secretion in the tear film leading to ocular surface changes and unstable tear film. MGD hampers day to day life activities as it leads to alteration of the lipids in tear film resulting in unstable tear film and causing inflammation on ocular surface and symptoms of eye irritation.

\section{Clinical Staging of MGD}

Considering the symptoms and signs of Meibomian gland dysfunction, grading of symptoms was divided into four stages as discussed in the International Workshop on Meibomian Gland Dysfunction and Management conducted in 2011.10

\section{Stage 1}

No symptoms of foreign body sensation, itching, or photophobia. Gland expressibility seen as:

- Minimum secretions: Greater than or equal to grade 2 to less than grade 4

- Expressibility: 1.

No ocular surface staining present.

\section{Stage 2}

Mild to moderate symptoms of foreign body sensation, itching, or photophobia.

Mild to moderate MGD clinical signs

- Thickening of the lid

- Mildly altered secretions: Greater than or equal to grade 4 to less than grade 8

- $\quad$ Expressibility: 1 .
Stage 3

Moderate symptoms of foreign body sensation, itching, or photophobia with restriction of day to day activities.

Moderate MGD clinical signs

- Increased plugging, blood vessel on lid

- Moderate altered secretions: Greater than or equal to grade 8 to less than grade 13

- Expressibility: 2.

Mild-to-moderate conjunctival and peripheral corneal staining

Stage 4

Marked symptoms of foreign body sensation, itching, or photophobia with definite limitations of activities.

Severe MGD clinical signs

- Increased lid margin features: dropout, displacement

- Severely altered secretions: Grade $\geq 13$

- Expressibility: 3.

Marked conjunctival and corneal staining, including central staining.

We wanted to study the corelation of chronic smoking with Meibomian gland dysfunction in Wardha, Maharashtra, India.

\section{METHODS}

This is a hospital based observational cross-sectional study which included total of 100 participants who were presented with symptoms of watering, ocular discomfort, irritation, burning sensation and giving history of smoking cigarette of more than 5 years in the outpatient department of ophthalmology in AVBRH, Sawangi. This study was conducted from May 2020 to October 2020. Passive smokers were excluded from the study. Approval was taken from ethical committee and study was conducted in accordance with Helsinki declaration. Informed consent was obtained from all the participants.

\section{Inclusion Criteria}

1. Subjects within age group of 20 - 80 years.

2. Cigarette smoking of more than 5 years.

\section{Exclusion Criteria}

1. Subjects with history of Stevens Johnson syndrome

2. Sjogren's syndrome

3. Past history of ocular trauma or chemical burns

4. Past ocular surgery

5. Infectious keratoconjunctivitis

6. Contact lens wearing

7. Ametropia more than 2 dioptres

8. Trachoma

9. Connective tissue disorder: Rheumatoid arthritis, SLE,

10. Pterygium

11. Dermatological disorders: psoriasis, acne rosacea / vulgaris 
The packet value was calculated by multiplying the mean number of packets of cigarettes smoked per day by the duration of smoking.

The same experienced ophthalmologist performed all the tests and examination. All the participants first underwent routine ophthalmological examination including visual acuity by Snellen's chart, slit lamp biomicroscopic anterior segment examination and fundus examination.

Grading of Meibomian gland expression: Assessed by slit lamp examination. The quality of expression will be graded according to the degree of opacity and viscosity on a $0-5$ scale-

1. Normal viscosity

2. Opaque with normal viscosity of the glands

3. Opaque with increased viscosity of the glands

4. Severe thickening (toothpaste like secretions) and

5. Fully blocked glands.

\section{Lid Margin Abnormality}

1. Absent

2. Present: vascular engorgement, plugged meibomian gland orifices.

\section{Grading of Symptoms}

1. Ocular symptoms like itching, discomfort, burning sensation

2. Any one of the sign-lid margin abnormality including irregular lid margin with displacement of mucocutaneous junction, engorgement of the vessels, plugged orifices of the gland.

3. Abnormal Meibomian gland expression.

Tear film functions are evaluated using two tests; TBUT and Schirmer's I test. For the TBUT, fluorescein papers were wet and touched to the lower fornix asking patients to blink 2 - 3 times to spread staining evenly on the ocular surface. On a silt lamp biomicroscope using a cobalt blue filter, examination of tear film was done. The time was calculated until the first dry point on the cornea was seen. Normal values were 15 - 35 seconds and value less than 10 seconds suggests an unstable tear film.

The Schirmer's test I was applied by instilling $0.5 \%$ proparacaine, and using standard filter paper (no. 41 Whatman Filter paper, $5 \times 35 \mathrm{~mm}$ long) which is placed on the lower fornix at the junction of the outer-third of the lower eyelid. The patient was instructed to blink normally. After 5 minutes, millimetres from the edge of the Schirmer test paper was calculated. Normal values of Schirmer's I test is more than $15 \mathrm{~mm}$. Values less than $5 \mathrm{~mm}$ is of severe kerato conjunctivitis sicca (KCS).

\section{Sample Size Calculation}

$\mathrm{N}=4 \mathrm{pq} / \mathrm{L}^{2}$

$\mathrm{P}=$ proportion of MGD $=64.6 \%$

$\mathrm{Q}=100-\mathrm{p}=100-64.6=35.4$

$\mathrm{L}=$ allowable error $=15 \%$ of $\mathrm{p}=15 \times 64.6 / 100=9.69$

$\mathrm{N}=4 \times 64.6 \times 35.6 / 9.692=97.42$

$=97.42$

$=100$ patients needed in the study

\section{RESULTS}

Smokers had decreased scores of TBUT, Schirmer's test and increase lid margin abnormality which suggests that MGD is more prevalent in smokers.

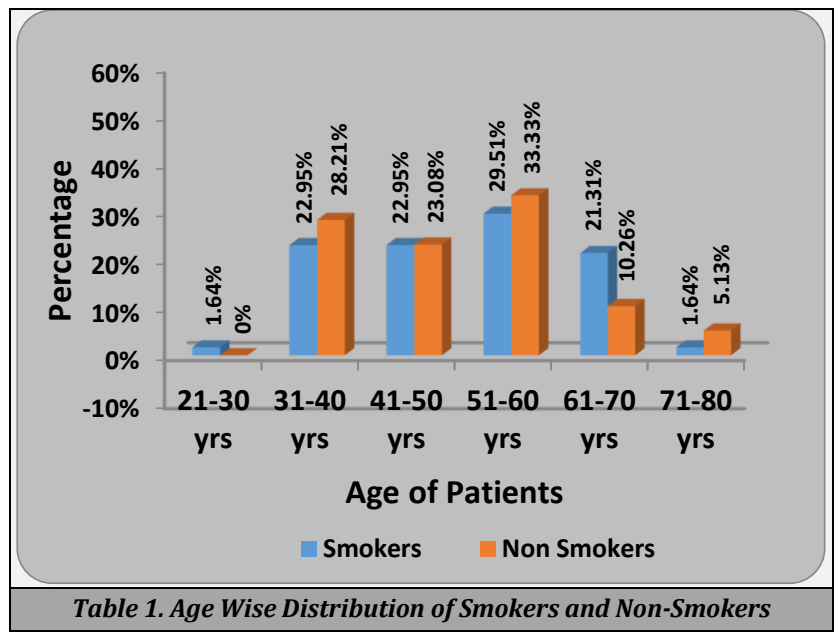

This is the age wise distribution of subjects in our study of total 100 subjects which included 61 smokers and 39 nonsmokers, age was found to be statistically insignificant with smoking. However, maximum smokers were found in age group of 51 - 60 years.

\begin{tabular}{|ccccc|}
\hline Gender & Smokers & Non-Smokers & Total & $\chi^{2}$-Value \\
Male & $50(79 \%)$ & $29(74.36 \%)$ & 79 & 0.83 \\
Female & $11(21 \%)$ & $10(25.64 \%)$ & 21 & $\mathrm{P}=0.36$, NS \\
Total & $\mathbf{6 1}(\mathbf{1 0 0} \%)$ & $\mathbf{3 9}(\mathbf{1 0 0} \%)$ & $\mathbf{1 0 0}$ & \\
\hline Table 2. Gender Wise Distribution of Smokers and Non-Smokers \\
\hline
\end{tabular}

Out of total smokers, 50 smokers were male as compared to 11 female smokers and gender wise distribution was statistically insignificant in our study.

\begin{tabular}{|ccccc|}
\hline & N & Mean & $\begin{array}{c}\text { Std. } \\
\text { Deviation }\end{array}$ & t-Value \\
Smokers & 61 & 7.45 & 2.03 & 6.66 \\
Non-smokers & 39 & 10.05 & 1.65 & $\mathrm{P}=0.0001, \mathrm{~S}$ \\
\hline \multicolumn{5}{c|}{$\begin{array}{c}\text { Table 3. Comparison of TBUT in Secs } \\
\text { among Smokers and Non-Smokers }\end{array}$} \\
\hline
\end{tabular}

Tear film break up time TBUT was found to be lower in 61 smokers with mean of 7.45 secs as compared to 39 nonsmokers with mean of 10.05 secs which is statistically significant.

\begin{tabular}{|cccccc|}
\hline & N & Mean & $\begin{array}{c}\text { Std. } \\
\text { Deviation }\end{array}$ & $\begin{array}{c}\text { Std. Error } \\
\text { Mean }\end{array}$ & t-Value \\
Smokers & 61 & 7.72 & 2.19 & \pm 0.28 & 6.72 \\
Non-smokers & 39 & 10.61 & 1.92 & \pm 0.30 & $\mathrm{P}=0.0001, \mathrm{~S}$ \\
\hline \multicolumn{5}{|c|}{$\begin{array}{c}\text { Table 4. Comparison of Schirmer I Test Score } \\
\text { among Smokers and Non-Smokers }\end{array}$} \\
\hline \multicolumn{7}{|c}{}
\end{tabular}

Schirmer's I test values were found to be lower in smokers with mean of $7.72 \mathrm{~mm}$ as compared to $10.61 \mathrm{~mm}$ in nonsmokers which is statistically significant.

Lid margin abnormality was found to be irregular in 29.51 $\%$ smokers as compared to $10.26 \%$ of non-smokers, thickened lid margin in $26.23 \%$ smokers as compared to $7.69 \%$ in nonsmoker and normal in $44.26 \%$ of smokers whereas, it was 
found to be normal in $82.05 \%$ of non-smokers which is statistically insignificant.

\begin{tabular}{|ccccc|}
\hline Lid Margin & Smokers & Non-Smokers & Total & $\chi^{2}$-Value \\
Irregular & $18(29.51 \%)$ & $4(10.26 \%)$ & 22 & \\
Thickened & $16(26.23 \%)$ & $3(7.69 \%)$ & 19 & 14.06 \\
Normal & $27(44.26 \%)$ & $32(82.05 \%)$ & 59 & $\mathrm{P}=0.001, \mathrm{NS}$ \\
Total & $\mathbf{6 1}(\mathbf{1 0 0} \%)$ & $\mathbf{3 9}(\mathbf{1 0 0} \%)$ & $\mathbf{1 0 0}$ & \\
\hline Table 5. Comparison of Lid Margin among Smokers and Non-Smokers \\
\hline
\end{tabular}

\begin{tabular}{|c|c|c|c|c|}
\hline Meibomitis & Smokers & Non-Smokers & Total & $\chi^{2}$-Value \\
\hline Present & $29(47.54 \%)$ & $5(12.82 \%)$ & 34 & \multirow{3}{*}{$\begin{array}{c}12.78 \\
P=0.0001, N S\end{array}$} \\
\hline Absent & $32(52.46 \%)$ & $34(87.18 \%)$ & 66 & \\
\hline Total & $61(100 \%)$ & $39(100 \%)$ & 100 & \\
\hline
\end{tabular}

Meibomitis was observed in $47.54 \%$ in smokers and 12.82 $\%$ of non-smokers which is found to be statistically insignificant. Statistical analysis is done by using descriptive and inferential statistics using chi square test and student's unpaired t test and software used in the analysis were SPSS 24.0 version and GraphPad Prism 7.0 version and $P<0.05$ was considered as level of significance.

\section{DISCUSSION}

Meibomian gland dysfunction is basically chronic altered secretion from the gland either quantitatively or qualitatively because of obstruction of terminal duct. It leads to thickening of the meibum secretion from the glands. Due to presence of terminal duct blockage of the glands which results in decreased amounts of lipids leading to uneven spreading of tears which suggests instability of the tear film. Various factors exacerbate like hyperosmolarity, increased growth of microorganisms, dryness of eye, and ocular surface inflammation leading to thickening, scarring of the gland orifices. Along with these ocular factors, various general factors such as old age, constant lens wearing, and hormonal imbalance, cigarette smoking can aggravate MGD.

Evaporative eye diseases are also exacerbated by various environmental factors. Cigarette smoking has been reported to have harmful effects on tear film instability and ocular surface abnormalities. Cigarette smoking directly causes tearing and instability of tear film. The pathophysiology of MGD is due to obstructive process caused by proliferation of epithelial cells of Meibomian duct. It interacts with increased flora of the gland and surrounding area and increased inflammatory mediators inside Meibomian gland and around the eyelid margin.

It is suggested that smoking causes inflammatory reactions which may be involved in association with smoking in MGD. Tobacco smoking causes disturbances in immune system and both the innate and adaptive immune responses are affected. Cigarette smoking leads to the production of proinflammatory cytokines - tumour necrosis factor (TNF alpha), interleukins (IL - 1, 6, 8). Many studies have shown molecular basis of inflammation as the effect of cigarette smoking on Meibomian gland. In this study, we reported lower TBUT scores and Schirmer's test values in smoker with MGD than non-smoker without MGD. Our results were in consistent with the previous studies which showed lower TBUT scores and Schirmer's test values in smokers than non-smokers.
Altinor et al. revealed that smoking causes damage to lipid layer of pre-corneal tear film by oxidative damage to lipids which causes dry eye symptoms.

Gabella merino et al. showed that TBUT scores and Schirmer's test values were not found to be significantly different in smokers with MGD as compared to non-smokers. Our study showed the clinical signs of MGD-thickening, irregularity of the eyelid margin, changes in the lid of blood vessels, presence of telangiectasia, plugging, blockage of the gland orifices, decrease in quantity and number of glands, changes in gland secretion, quality, and viscosity.

Visco et al. reported that past smoking was a risk factor for MGD and our study showed that chronic cigarette smoking is correlated with MGD. This study revealed that the lid margin abnormality and meibum were found to be higher in smokers as compared to non-smokers in MGD patients. Additionally, packet value was found to be correlated with lid margin abnormality, TBUT scores, Schirmer's test values and meibum in smokers with MGD.

A study by Wang et al. showed that upon comparison with the MGD patients without smoking, the MGD patients with smoking had increased values of lid margin abnormality and meibum whereas TBUT test and Schirmer's I test was found to be insignificant in smokers with MGD. Schirmer's test was found to be $6.29 \pm 0.30$ as compared to our study Schirmer's test I is found to be $7.72 \pm 0.26$ which is statistically significant and found to be lower in smokers as compared to nonsmokers. In our study we found lower values of TBUT test and Schirmersu test in smokers with MGD along with increased scores of lid margin abnormality. And the rate of tear production and evaporation was increased more in MGD than non MGD group.

Tear break up time in gland drop out group was found to be $5.68 \pm 0.68$ as compared to our study $7.45 \pm 0.26$. Schimazaki et al. showed that MGD group had higher score of staining with fluorescein dye as compared with that of non MGD group.

\section{CONCLUSIONS}

Meibomian gland dysfunction is a major cause of evaporative eye disease which is associated with cigarette smoking leading to ocular discomfort and lid abnormalities. Chronic smoking is associated with Meibomian gland dysfunction causing lid abnormalities, inflammation, and unstable tear film with decreased TBUT and Schirmer's test.

Data sharing statement provided by the authors is available with the full text of this article at jemds.com.

Financial or other competing interests: None.

Disclosure forms provided by the authors are available with the full text of this article at jemds.com.

\section{REFERENCES}

[1] Bartecchi CE, MacKenzie TD, Schrier RW. The human costs of tobacco use (1). N Engl J Med 1994;330(13):90712. 
[2] Bartecchi CE, MacKenzie TD, Schrier RW. The global tobacco epidemic. Sci Am 1995;272(5):44-51.

[3] Lee KS, Park CY, Meng KH, et al. The association of cigarette smoking and alcohol consumption with other cardiovascular risk factors in men from Seoul, Korea. Ann Epidemiol 1998;8(1):31-8.

[4] Loewenstein A, Rosner M, Solberg Y, et al. Passive smoking and its influence on the eye. Harefuah 2000;139(7-8):280-1.

[5] Solberg Y, Rosner M, Belkin M. The association between cigarette smoking and ocular diseases. Surv Ophthalmol 1998;42(6):535-47.

[6] Sayin N, Kara N, Pekel G, et al. Effects of chronic smoking on central corneal thickness, endothelial cell and dry eye parameters. Cutan Ocul Toxicol 2014;33(3):201-5.
[7] Mathers WD, Lane JA. Meibomian gland lipids, evaporation, and tear film stability. Adv Exp Med Biol 1998;438:349-60.

[8] Jester JV, Nicolaides N, Smith RE. Meibomian gland studies: histologic and ultrastructural investigations. Invest Ophthalmol Vis Sci 1981;20(4):537-47.

[9] Mishima S, Maurice DM. The oily layer of the tear film and evaporation from the corneal surface. Exp Eye Res 1961;1:39-45.

[10] Guliani BP, Bhalla A, Naik MP. Association of the severity of meibomian gland dysfunction with dyslipidemia in Indian population. Indian J Ophthalmol 2018;66(10):1411-6. 